\title{
A comparative study of the effects of concentrated growth factors in two different forms on osteogenesis in vitro
}

\author{
LIPING WANG ${ }^{*}$, MIANJIA WAN*, ZHENGMAO LI, NINGYING ZHONG, \\ DONGLIANG LIANG and LINHU GE \\ Key Laboratory of Oral Medicine, Guangzhou Institute of Oral Disease, \\ Affiliated Stomatology Hospital of Guangzhou Medical University, \\ Guangzhou, Guangdong 510140, P.R. China
}

Received December 23, 2018; Accepted April 24, 2019

DOI: $10.3892 / \mathrm{mmr} .2019 .10313$

\begin{abstract}
Extending the release cycle of growth factors to match the cycle of bone remodeling is difficult. When using concentrated growth factors (CGFs), the release of growth factors is excessively rapid. In the present study, CGF samples were prepared by centrifugation. CGF samples were then lyophilized and grinded into a powder, which was termed freeze-dried CGF. The freeze-dried CGF samples were mixed with chitosan-alginate composite hydrogels, and the mixture was lyophilized. The result was a chitosan-alginate composite CGF membrane, which was called sustained-release CGF. This study investigated whether freeze-dried CGF in a chitosan-alginate composite gel can release CGF steadily to achieve effective osteogenesis. The proliferation and osteogenic expression of MC3T3-E1 cells induced by the supernatants from incubation with freeze-dried CGF and sustained-release CGF were evaluated. The concentrations of the growth factors, transforming growth factor $\beta 1$ (TGF- $\beta 1$ ), insulin-like growth factor-1 (IGF-1), platelet-derived growth factor-AB (PDGF-AB) and vascular endothelial growth factor (VEGF), in these two experimental groups at different times were determined by ELISA kits. The freeze-dried CGF showed better osteogenic performance than the sustained-release CGF in the early stages. At later stages, the sustained-release CGF had significant advantages over freeze-dried CGF in terms of promoting osteogenic mineralization. By characterizing the biologic properties of the CGF in the two different forms
\end{abstract}

Correspondence to: Professor Linhu Ge, Key Laboratory of Oral Medicine, Guangzhou Institute of Oral Disease, Affiliated Stomatology Hospital of Guangzhou Medical University, 39 Huangsha Avenue, Liwan, Guangzhou, Guangdong 510140, P.R. China

E-mail: gygelinhu@163.com

*Contributed equally

Key words: concentrated growth factors, CGF, freeze-dried CGF, sustained-release CGF, osteogenesis, in vitro in vitro, we obtained a better understanding of their clinical effects.

\section{Introduction}

Bone regeneration is mediated by a wide range of intracellular and extracellular events. In clinical settings, platelet concentrates can be used to optimize the healing of hard and soft tissues.

Platelet gel, which was first described in 1997, is derived from collected autologous blood and formed by combining platelet-rich plasma (PRP), thrombin, and calcium chloride (1). PRP is an autologous source of platelet-derived growth factor (PDGF) and transforming growth factor $\beta$ (TGF- $\beta$ ) that is obtained by sequestering and concentrating platelets by gradient density centrifugation (2).

In 2001, platelet-rich fibrin (PRF) was discovered in France by J. Choukroun using an innovative method that required neither anticoagulants nor coagulation factors. PRF, which is called the second-generation platelet concentrate, has been shown to contain more growth factors than traditionally prepared PRP (3).

Recently, numerous techniques using platelet concentrates have been developed to obtain different ratios of platelets, growth factors, leukocytes and fibrin matrices (4). Concentrated growth factors (CGFs) are the PRF derivatives developed by Sacco in 2006 (5). CGFs are produced by centrifuging blood samples with a special centrifuge device (Medifuge, Silfradent Srl, Italy). Nevertheless, CGFs contain a much larger, denser and richer growth factor fibrin matrix than PRF $(5,6)$.

CGFs are now widely used to shorten the interval between bone graft placement and implant insertion and increase the success rate of bone grafting and implant therapy. Many articles have been published on the application of CGFs in the dental and maxillofacial field (7-9).

When using concentrated growth factors (CGFs), the release of growth factors is excessively rapid. He et al (10) showed that the three-dimensional fiber network scaffold of CGFs can help release growth factors slowly for at least 7-10 days. However, bone healing is a long and complex process. Most of these studies focused on the application of CGFs in the clinic. Extending the release cycle of growth factors to match 
the cycle of bone remodeling is difficult, and few reports have mentioned this issue.

Chitosan, also known as soluble chitin, is a natural polysaccharide cellulose that is nontoxic, biocompatible, biodegradable, and widely found in insect, crustacean shells and fungal cell walls $(11,12)$. Its preparation is simple, its source is rich, and its hydrophilicity is strong. Chitosan can be biodegradable by in vivo lysozyme, pepsin, and other enzymes. The degradation products are nontoxic and can be completely absorbed by the organisms (13). Chitosan has a good film-forming property, and the film has good biological compatibility and permeability. Thus, chitosan has important development and research value for sustained-release drugs and targeted drug delivery, and it is often used as the vehicle for the sustained release of drugs. Sustained-release drugs are released slowly over a long period of time to achieve sustained administration (14). Chitosan can be used to prepare microspheres of different sizes. The combination of microspheres and drugs can avoid the use of organic solvents and prevent the denaturation of antigenic proteins (15). Chitosan has a unique polycationic property.

Sodium alginate (SA), a negatively charged biopolysaccharide, is extracted from brown seaweed. SA has also been widely used for hemostatic applications because of its excellent abilities to enhance the adhesion between composite materials and wounds, to improve material plasticity through its high water absorption rate and to easily form a viscous colloidal solution $(16,17)$.

However, while the positive charge of chitosan promotes erythrocyte adhesion, fibrinogen adsorption, and platelet adhesion/stimulation, it also inhibits the activation of the contact system (18). Chitosan interacts with sodium alginate (polyanion) by electrostatic interaction, which can improve microcapsule stability and drug loading and adjust the drug release rate (19).

Therefore, we aimed to preserve CGFs for storage by freeze-drying without additives. Furthermore, we investigated whether the freeze-dried CGFs in a chitosan-alginate composite gel can release CGFs steadily to achieve effective osteogenesis.

\section{Materials and methods}

Blood sample centrifugation. Vein blood samples were collected from 10 healthy volunteers (4 females and 6 males), who were nonsmokers between 20 and 30 years of age.

CGFs were produced as follows: $5 \mathrm{ml}$ of blood was drawn from the arm vein in blood collection tubes without anticoagulant solution, with 2 tubes used for each collection between October 2017 and December 2018. These tubes were immediately centrifuged in a special machine (Medifuge MF200, Silfradent Srl, Italy). At the end of the process, there were three blood fractions. The middle part (CGF) as shown in Fig. 1A (fibrin-rich gel with aggregated platelets and CGFs) was removed.

Lyophilization of CGFs. CGFs were pre-frozen at $-80^{\circ} \mathrm{C}$ for $12 \mathrm{~h}$ and then lyophilized for $24 \mathrm{~h}$ using a freeze dryer (Martin Christ Freeze Dryers GmbH, Germany). After freeze-drying, samples were ground into a powder, which we called freeze-dried CGF. Then, $0.02 \mathrm{~g} / \mathrm{ml}$ alginate was mixed with the CGF powder, and $0.02 \mathrm{~g} / \mathrm{ml}$ chitosan was mixed with the alginate-CGF powder composite hydrogels, then, the composite CGF hydrogels were lyophilized again. Finally, the chitosan-alginate composite CGF membrane, which was called sustained release CGFs was obtained (Fig. 1). Both types of CGFs were stored at $-4^{\circ} \mathrm{C}$ for one month.

Cell culture. The murine-derived cell line MC3T3-E1 was used in this study. MC3T3-E1 cell cultures were maintained in minimum essential medium (MEM, Gibco; Thermo Fisher Scientific, Inc.) supplemented with $10 \%$ fetal bovine serum (FBS, Gibco; Thermo Fisher Scientific, Inc.), and 1\% (v/v) penicillin/streptomycin (Gibco; Thermo Fisher Scientific, Inc.) at $37^{\circ} \mathrm{C}$ in a humidified $\mathrm{CO}_{2}$ incubator. Cells at approximately $80 \%$ confluence, were passaged by trypsin digestion and expanded through two passages before being used for the study.

\section{Material cytotoxicity test}

Cell climbing of chitosan-alginate composite hydrogels. The prepared chitosan-alginate composite gel was spread on a cell slide, which was placed in a 6-well plate, air dried, sterilized by ultraviolet light for 1 day, seeded with 5,000 cells per well, and fixed with paraformaldehyde after 3 days. After climbing, air drying, and spraying with gold, the cell morphology was observed under a scanning electron microscope (Hitachi S-3400N; Hitachi, Ltd.).

Live and dead assays of sustained-release CGFs. The sustained-release CGFs were spread on a 48-well plate, which was seeded with $1 \times 10^{4}$ cells per well. Cell viability was evaluated with confocal microscopy after staining with calcein AM and propidium iodide (PI) (Invitrogen; Thermo Fisher Scientific, Inc.) at days 3 and 5 after seeding. Samples were incubated for $20 \mathrm{~min}$ at $37^{\circ} \mathrm{C}$ with the calcein solution in culture medium ( $1 \mu \mathrm{l}$ of calcein per ml of MEM), washed in phosphate-buffered saline (PBS), exposed to the second staining solution with PI in PBS (100 $\mu \mathrm{l}$ of PI pe ml of PBS), double washed in PBS and immediately visualized with a confocal microscope (Leica Microsystems CMS GmbH).

Cell proliferation and metabolic activity of the two forms of CGFs. CGFs were placed in a $15-\mathrm{ml}$ centrifuge tube, and $5 \mathrm{ml}$ of culture medium was added. This sample was stored in a refrigerator at $4^{\circ} \mathrm{C}$ for $24 \mathrm{~h}$. The incubation solution was a gradient series of concentrations (20, 40, 60, 80 and 100\%). MC3T3-E1 cells were seeded in a 96-well tissue culture plate at a density of 3,000 cells per well. After $24 \mathrm{~h}$ of incubation, the culture medium was removed and replaced with $200 \mu \mathrm{l}$ of different concentrations of CGF extract containing 10\% FBS. At days 1, 3 and 5, Cell Counting Kit-8 (CCK-8) assay was applied to determine the overall proliferation. The absorbance [optical density (OD) value at $450 \mathrm{~nm}$ ] was read by a microplate reader (Thermo Scientific, Inc.). The most suitable concentrations of the CGFs were screened based on the results of the MC3T3-E1 proliferation study and were used in the following experiments.

A control group, a freeze-dried CGF group, a sustained-release CGF group, and a blank chitosan-alginate composite hydrogel group were used. MC3T3-E1 cells were 
A

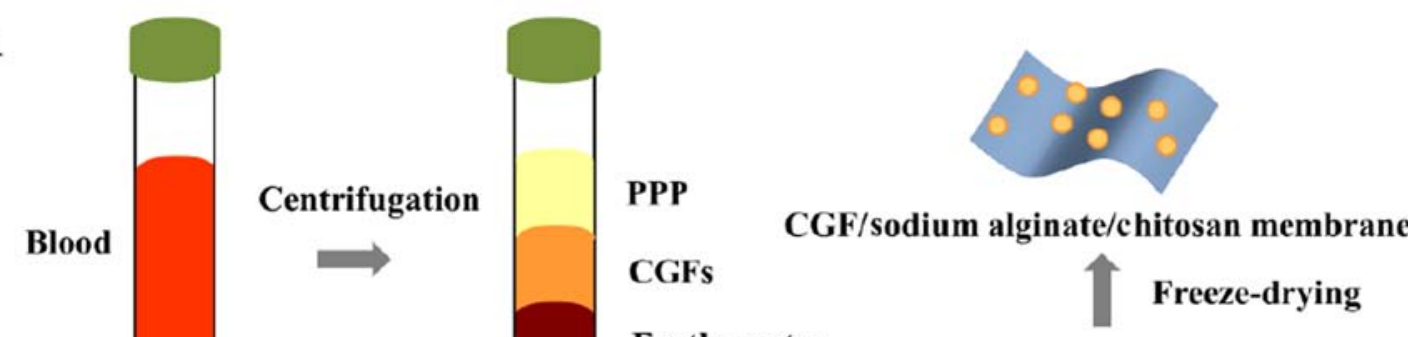

Erythrocytes

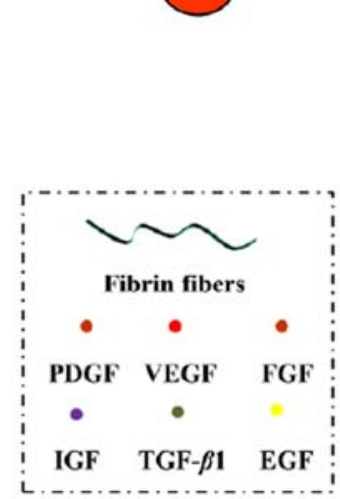

CGF contains

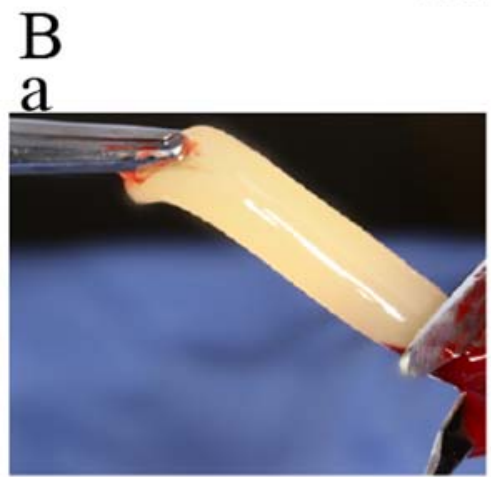

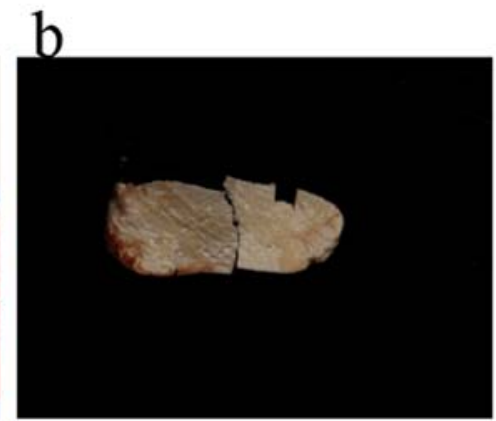

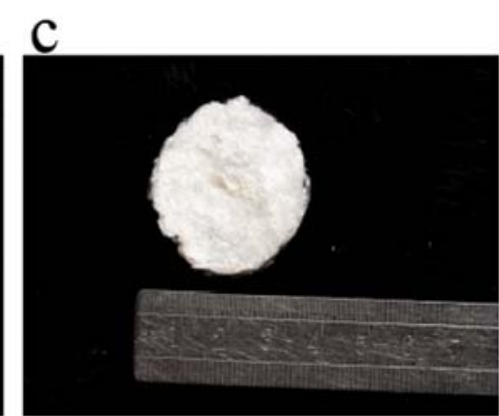

Figure 1. (A) Preparation schematic of the sustained-release CGFs. (B) Three different statuses of CGFs. (a) CGFs that we use in the clinic. (b) Freeze-dried CGFs before being ground. (c) Sustained-release CGFs. CGFs, concentrated growth factors; PPP, platelet poor plasma; PDFG, platelet-derived growth factor; VEGF, vascular endothelial growth factor; FGF, fibroblast growth factor; IGF, insulin-like growth factor; TGF- $\beta 1$, transforming growth factor $\beta 1$; EGF, epidermal growth factor.

seeded in a 96-well tissue culture plate at a density of 3,000 cells per well. After $24 \mathrm{~h}$ of incubation, the culture medium was removed and replaced with $200 \mu$ of different extracts containing 10\% FBS. On days 1-6, a CCK-8 assay was applied to determine the overall proliferation.

Alkaline phosphatase (ALP) quantification. MC3T3-E1 cells were seeded in a 24-well tissue culture plate at a density of $2.5 \times 10^{4}$ cells per well. After $24 \mathrm{~h}$, the corresponding reagents were added to the experimental groups. The cells were lysed with Triton X-100 for 4, 7 and 14 days, and the total protein concentration was measured using a BCA assay kit (Beyotime, China). The ALP activity was measured using an ALP assay kit (Nanjing Jiancheng Biotech, China).

Cells were seeded in a 48 -well tissue culture plate at a density of $2 \times 10^{4}$ cells per well. After $24 \mathrm{~h}$, the corresponding reagents were added to the experimental group. After 14 and 21 days, the cells were stained with a BCIP/NBT alkaline phosphatase colorimetric kit (Beyotime).
Alizarin Red S staining. To identify the mineralization nodules, Alizarin Red S (ARS; Sigma-Aldrich; Merck KGaA) staining was performed after the MC3T3-E1 cells were seeded at a density of $2 \times 10^{4}$ cells per well and grew for 21 days in conditioned or control media without osteogenic supplements. After gently rinsing with $\mathrm{ddH}_{2} \mathrm{O}$, the cells were stained in a solution of $2 \%$ ARS at pH 4.1 for 20 min and then washed with $\mathrm{ddH}_{2} \mathrm{O}$. The samples were air-dried, and images were captured under a light microscope (magnification, $\mathrm{x} 6$ ). Additionally, the bound dye was dissolved with $10 \%$ cetylpyridinium chloride, and the ARS in the samples was quantified by measuring the absorbance at $562 \mathrm{~nm}$.

Bone-related gene expression. For the detection of bone-related genes (Table I), i.e., ALP, osteopontin $(O P N)$, osteoclastogenesis inhibitory factor $(O P G)$, collagen type I $(C O L 1)$, osteocalcin $(O G)$, Runx2 and bone sialoprotein $(B S P)$, MC3T3-E1 cells were plated at a density of $1 \times 10^{4}$ cells per well in separate 6 -well plates. After $24 \mathrm{~h}$ of incubation, the 
Table I. Primer pairs used in reverse transcription-quantitative quantitative PCR.

Genes Primer sequences

GAPDH Forward: 5'-AAGAAGGTGGTGAAGCAGG-3' Reverse: 5'-GAAGGTGGAAGAGTGGGAGT-3'

$O P G \quad$ Forward: 5'-AGTTTTGGGAAAGTGGGATGT-3' Reverse: 5'-GCTACTCAGTTTATGGAGGATCA-3'

$O G \quad$ Forward: 5'-GTAACGAGTGTCATTAGCCTTG-3' Reverse: 5'-ATAACGACCTGGAATCTGTGC-3'

Runx2 Forward: 5'-TCAGCGTCAACACCATCATTC-3' Reverse: 5'-CCAGACCAGCAGCACTCCATA-3'

BSP Forward: 5'-CAAAAGTCTGTGCTTGGGGTG-3' Reverse: 5'-GGAAAACAATGAAGATTCTGAGGG-3'

OPN Forward: 5'-CCTTAGACTCACCGCTCTTCAT-3' Reverse: 5'-TTCACTCCAATCGTCCCTACA-3'

ALP Forward: 5'- CAGTTCGTATTCCACATCAGTTC-3' Reverse: 5'-CAAGGACATCGCATATCAGCT-3'

COLIAl Forward: 5'-AGAACAGCGTGGCCT-3' Reverse: 5'-TCCGGTGTGACTCGT-3'

GAPDH, glyceraldehyde-3-phosphate dehydrogenase; $O P G$, osteoprotegerin; $O G$, osteocalcin; Runx2, runt-related transcription factor 2; $B S P$, bone sialoprotein; $O P N$, osteopontin; $A L P$, alkaline phosphatase; COL1A1, collagen type I $\alpha 1$.

culture medium was removed and replaced with conditioned medium or control medium. Total RNA from all groups was extracted using TRIzol reagent after 7, 14, 21 and 28 days of culture and analyzed by reverse transcription-quantitative (RT-q) PCR.

ELISA quantification. The freeze-dried CGFs and sustained-release CGFs were placed in a $15-\mathrm{ml}$ centrifuge tube, and $5 \mathrm{ml}$ of PBS was added. The samples were stored in a refrigerator at $4^{\circ} \mathrm{C}$. We took $1 \mathrm{ml}$ extract out to perform the ELISA and added $1 \mathrm{ml}$ fresh PBS to the tube to maintain a total volume of $5 \mathrm{ml}$ at each time point. When all samples were collected, quantifications of transforming growth factor $\beta 1$ (TGF- $\beta 1$ ), insulin-like growth factor-1 (IGF-1), platelet-derived growth factor-AB (PDGF-AB), vascular endothelial growth factor (VEGF), and thrombospondin-1 (TSP-1) were performed using commercially available ELISA kits (Cusabio, Biotech Co, Ltd., Wuhan, China). For each molecule and each experimental period, the means and standard deviations were calculated. Finally, for each tested molecule, the total released amounts were calculated, and these results were then compared to the initial amount forcibly extracted from the freeze-dried CGFs and the sustained-release CGFs.

Statistical analysis. All analyses were performed using SPSS 25 software (IBM Corp., Armonk, NY, USA). All experiments were performed at least in three independent repeats. All data are shown as the mean and standard deviation (SD) and were analyzed using one-way ANOVA or a nonparametric test followed by the least significant difference post hoc test. The levels of significance were set at ${ }^{*} \mathrm{P}<0.05,{ }^{* *} \mathrm{P}<0.01$ and ${ }^{* * * *} \mathrm{P}<0.001$ (as indicated in the figures and legends with the corresponding symbols).

\section{Results}

Characterization of the three types of CGFs. The architecture and morphology of the CGFs, freeze-dried CGFs, and sustained-release CGFs are shown in Fig. 1B. The surface morphology of freeze-dried CGFs and powdered freeze-dried CGFs were examined via scanning electron microscopy (SEM) (Fig. 2A). The freeze-dried CGF powder, hereafter called freeze-dried CGF, was used for the following in vitro studies.

Evaluation of cell bioactivity with the sustained-release CGFs. The cell climbing results with the chitosan-alginate composite hydrogels are presented in Fig. 2B. There were many cell nuclei highlighting the hydrogels (Fig. 2B-b). At the junction of the gel, MC3T3-E1 cells (Fig. 2B-c) could be observed, which suggested that the chitosan-alginate composite hydrogels had excellent biocompatibility and was non-toxic to the MC3T3-E1 cells.

The live and dead assays of the sustained-release CGFs and blank chitosan-alginate gel on day 5 are shown in Fig. 3. For this assay, we observed a much greater abundance of living MC3T3-E1 cells in the sustained-release CGF group than that in the blank gel group. Compared to the cells in the blank gel group, the MC3T3-E1 cells in the sustained-release CGF group adhered more tightly. This finding suggested that the sustained-release CGFs had an obvious ability to promote cell proliferation and adhesion.

Effect of the two forms of CGFs on osteoblast proliferation. The proliferation of MC3T3-E1 cells under different concentrations of CGFs was assessed using CCK- 8 assay. The analysis showed that the metabolic activity of MC3T3-E1 cells at different concentrations of CGFs had significant differences (Fig. 4). The medium with incubation solution extracts (20\%) was used for the following in vitro studies.

MC3T3-E1 cells grew well in both the culture medium and the medium with a series of material extracts. The CCK- 8 analysis showed that the overall metabolic activity of most groups with $20 \%$ material extracts increased in a time-dependent manner (Fig. 5). Compared with that of the control group, the cell proliferation rates in the freeze-dried CGF group and the sustained-release CGF group were significantly increased on day $6(\mathrm{P}<0.001)$. In contrast, the material group did not exhibit a difference in cell proliferation throughout the time period. This finding suggested that the simple chitosan-alginate composite hydrogels could not promote MC3T3-E1 cell proliferation and that only sustained-release CGFs, which combined chitosan-alginate composite hydrogels and CGFs, could promote cell proliferation.

Effect of the two forms of CGFs on ALP activity. ALP is central to osteogenesis. The level and activity of ALP are considered early osteogenic markers, particularly in in vitro experiments, as predictors of bone maturation and mineralization (20). The 


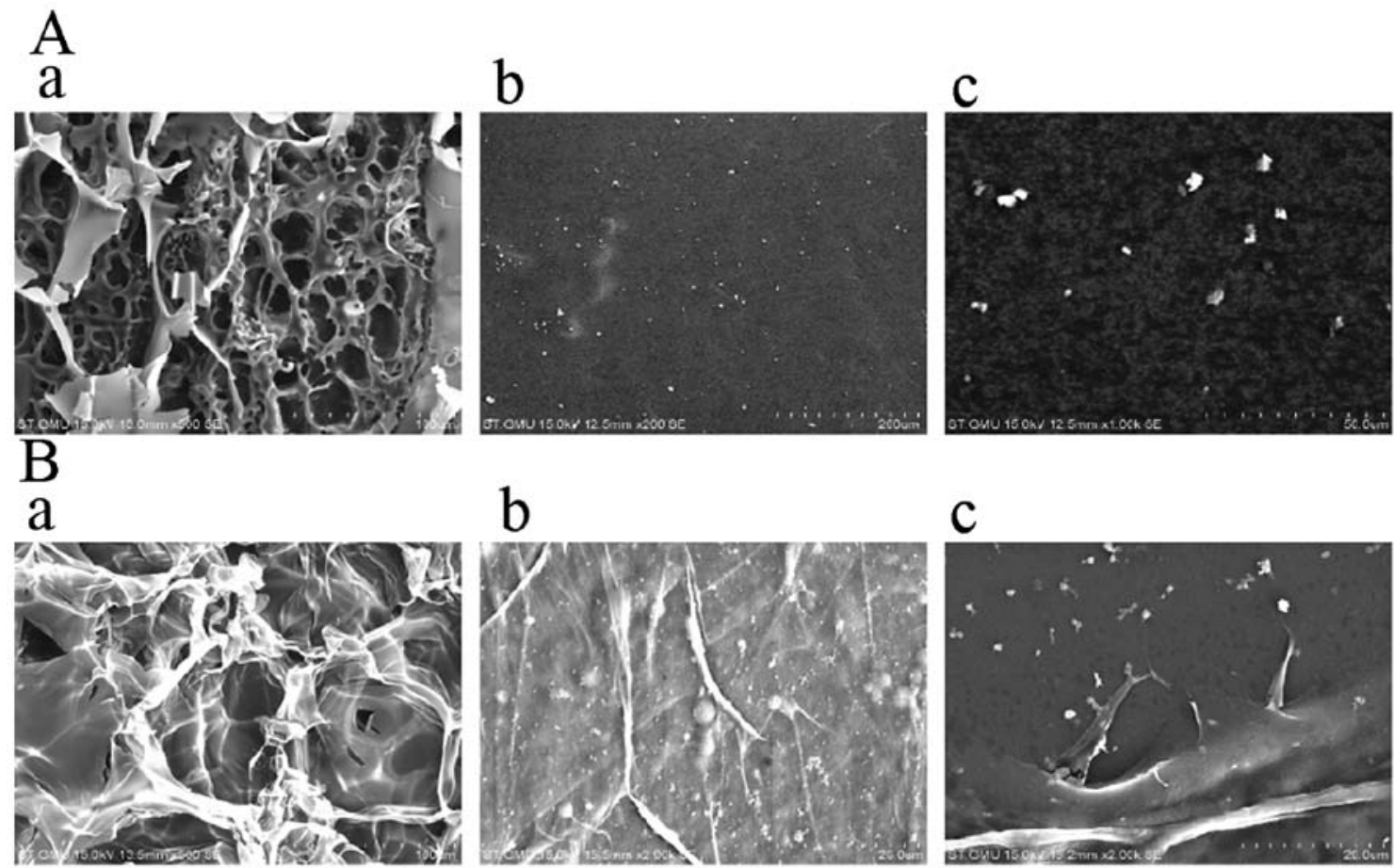

Figure 2. (A) Freeze-dried CGFs and (B) freeze-dried chitosan-alginate gel under a scanning electron microscope. (A) (a-c) Freeze-dried CGFs before being ground consisted of the freeze-dried CGF powder. (B) (a) Structure of the freeze-dried chitosan-alginate gel. (b) Upon the cell climbing, the chitosan-alginate gel area was covered with MC3T3-E1 cells. (c) At the junction of the gel, a MC3T3-E1 cell crawling onto the gel was noted. CGFs, concentrated growth factors.
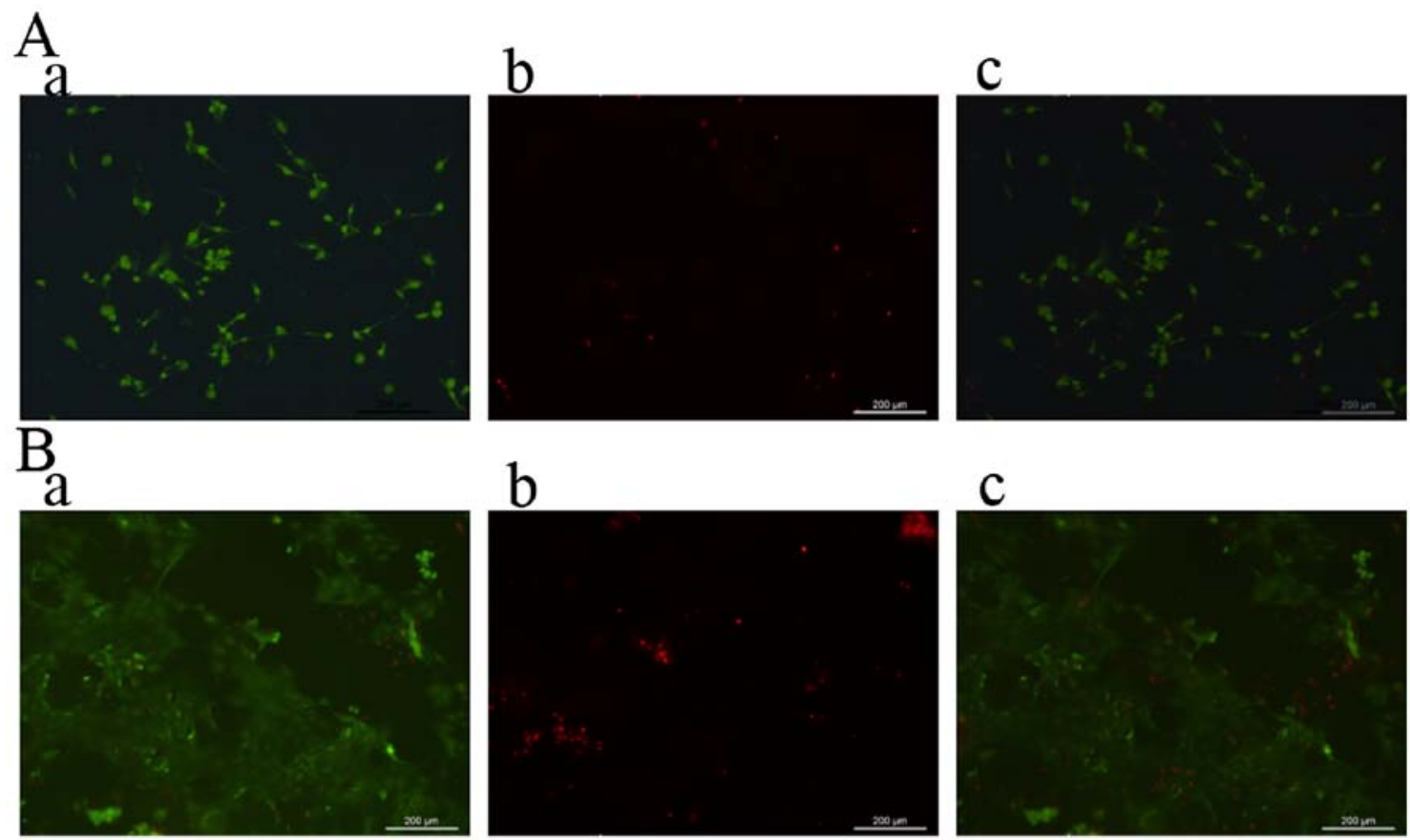

Figure 3. The live and dead assay of sustained-release CGFs and blank chitosan-alginate gel at day 5. (A) The blank chitosan-alginate gel group. (B) The sustained-release CGF group. (A and B) (a) Those cells dyed green were living MC3T3-E1 cells. (b) Those cells stained red were dead MC3T3-E1 cells. (c) Images a and b were superimposed, respectively. CGFs, concentrated growth factors.

results of ALP staining (Fig. 6A) in the (a) control group, (b) freeze-dried CGF group and (c) sustained-release CGF group are shown on day 14 of culture. The ALP activity in the MC3T3-E1 cells at day 14 of culture for the different groups is shown in Fig. 6A-d. ALP production increased with incubation time in all groups. At 14 days of incubation, the freeze-dried
CGF group had significantly higher ALP activity than the other groups (Fig. 6A-d).

The results of ALP staining of MC3T3-E1 cells at 14 days are shown in Fig. 6A. At 21 days (Fig. 6C, first row), the ALP staining of the MC3T3-E1 cells in the sustained-release CGF group (Fig. 6C-c-1) was clearer than that in the other 


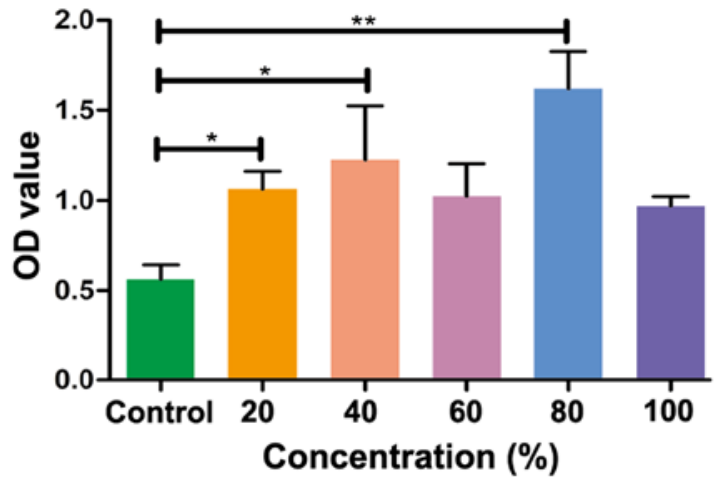

Figure 4. Proliferation of the MC3T3-E1 cells cultured with different concentrations of CGFs as detected by CCK- 8 assay. ${ }^{*} \mathrm{P}<0.05,{ }^{* *} \mathrm{P}<0.01$. CGFs, concentrated growth factors.
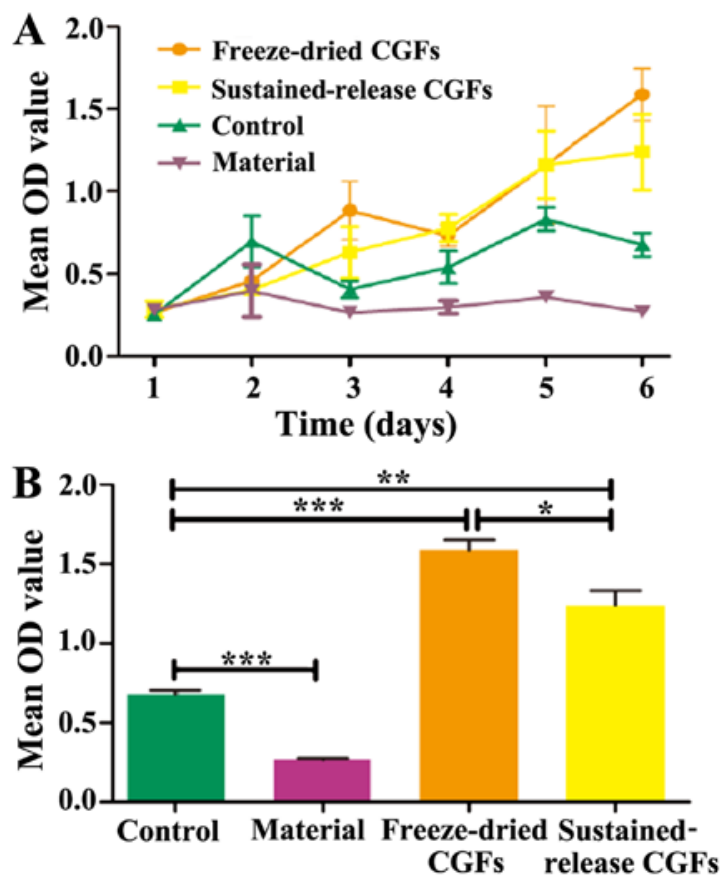

Figure 5. Effects of (A and B) freeze-dried CGFs, sustained release CGFs and the material on MC3T3-E1 cell proliferation were assessed using the Cell Counting Kit- $8 .{ }^{*} \mathrm{P}<0.05,{ }^{* *} \mathrm{P}<0.01,{ }^{* * *} \mathrm{P}<0.001$. The material group is the blank Chitosan-alginate composite gel. CGFs, concentrated growth factors.

groups (Fig. 6C-a-1 and -b-1). This finding suggested that, between 14 and 21 days, the expression of ALP in the sustained-release CGF group gradually increased, possibly due to the slow release of growth factors in the sustained release CGFs.

Effect of the two forms of CGFs on mineralization capability. The effects of the two forms of CGFs on MC3T3-E1 cell mineralization capability were assessed using ARS staining. As shown in Fig. 6B [(a) control group, (b) freeze-dried CGF group and (c) sustained-release CGF group], the amount of mineralization nodules in the sustained-release CGF group was obviously higher than that in the other group (Fig. 6B-d). When we performed ALP staining, we found that, in the location with ALP staining, there was a translucent circular plaque in the middle, which we suspected was a mineralized nodule.
To understand the relationship between ALP and mineralization, we performed ALP staining and ARS staining in the same cell frame (Fig. 6C) (Fig. 6C-a-2 to c-2; second row). The double staining results suggested that the sustained-release CGFs (Fig. 6C-c-2) had an obvious ability to promote ALP expression and osteogenic mineralization under long-term observation.

Assessment of osteogenesis-associated genes. The expression of genes associated with MC3T3-E1 were evaluated by RT-qPCR on days 7, 14, 21 and 28. As shown in Fig. 7, $B S P$ (Fig. 7A), $O G$ (Fig. 7B), $O P G$ (Fig. 7C), and $O P N$ (Fig. 7D) showed more significant upregulation on day 7 in the freeze-dried CGFs group when compared with the other groups. (Fig. 7E) A significant upregulation of the gene expression of COL1A1 occurred on day 14 in the freeze-dried CGF group. (Fig. 7G) ALP was found to be significantly upregulated on day 28 in the sustained-release CGFs. (Fig. 7F) The gene expression of Runx 2 showed significant upregulation on day 21 .

Growth factor release analysis. Significant amounts of each growth factors were found at each experimental time point, even 30 days after the production of the freeze-dried CGFs and the sustained-release CGFs (Fig. 8). Moreover, these growth factors and TSP-1 resulted in a significantly rapid declined in the freeze-dried group before day 7 . This finding could also explain the very high expression of osteogenic genes $(B S P$, $O G, O P N, O P G)$ on day 7 for the the sustained release CGF group. The sustained-release group maintained the release of growth factors at the same concentration.

\section{Discussion}

CGFs have recently received a great deal of interest as the growth factors in CGFs can be locally released into tissue to enhance wound healing.

Seventy percent of the growth factors in PRP are released within $10 \mathrm{~min}$, and almost $100 \%$ are released within $1 \mathrm{~h}$ after activation (21,22). Dohan Ehrenfest et al (23) found that platelet-rich fibrin (PRF) can release growth factors slowly for at least one week, which is attributed to its fiber network scaffold. The study also suggested that the release rate is dependent on the environment surrounding the PRF (24). CGFs can release growth factors slowly for at least 7-10 days. The new bone formation (NBF) rate around an implant treated with CGFs is higher than that treated with PRF (25).

Currently, the major applications of CGFs in damaged tissue utilize its gelatin form. We lyophilized and ground the CGFs into a powder. Then, we integrated chitosan-alginate composite gel, freeze-dried CGFs into a membrane and evaluated the physical and osteogenic capacities of this membrane. In addition, through the quantitative detection of growth factors released from freeze-dried CGFs and sustained release CGFs, we explored the feasibility of using this composite gel as a carrier to achieve long-term sustained-release of CGFs.

We first assessed the biosafety of the chitosan-alginate composite gel via cell climbing and cell proliferation assays (Figs. 2 and 5).The data indicated that the gel was 

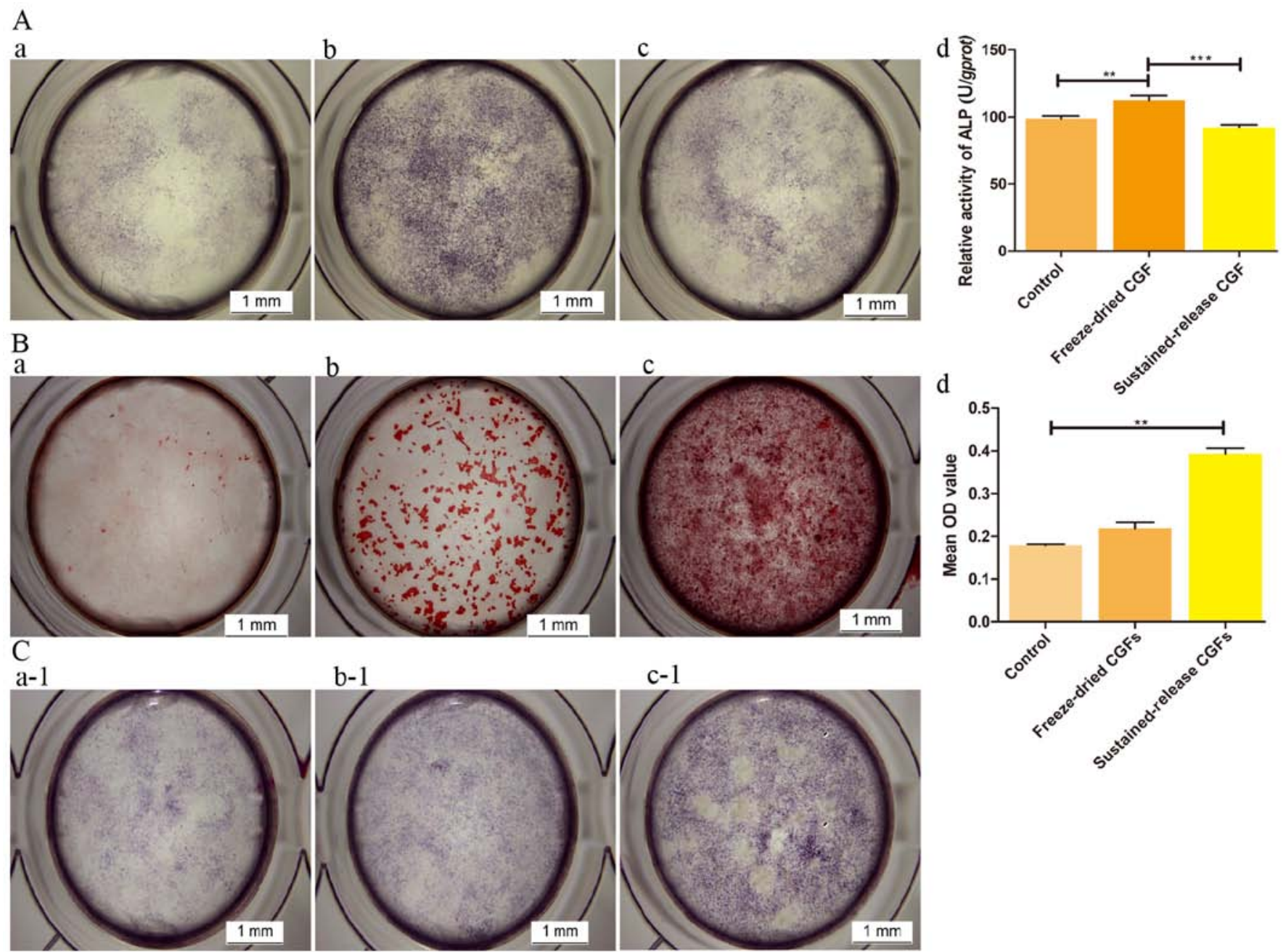

b-1
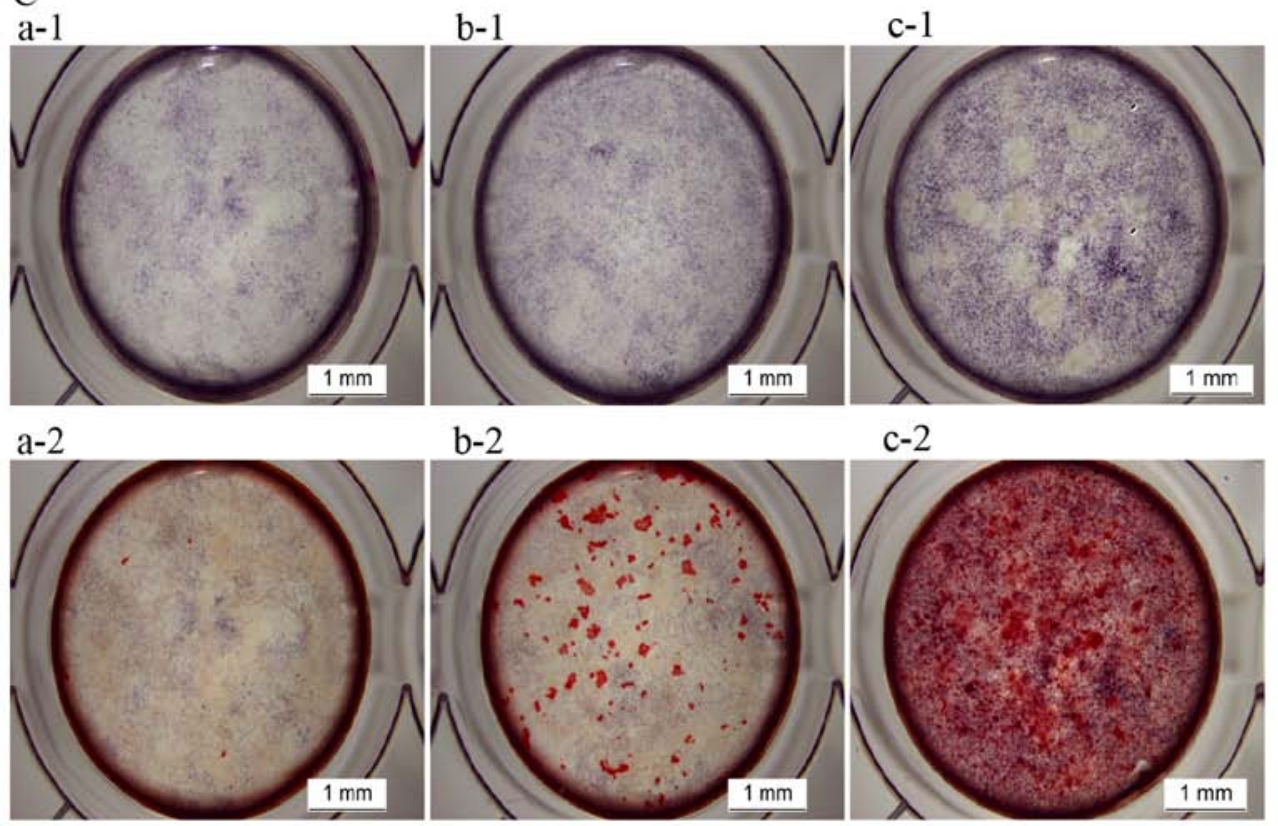

Figure 6. (A) (a-c) Results of alkaline phosphatase (ALP) staining in the different groups after stimulation of MC3T3-E1 cells for 14 days. (d) Expression of ALP protein for the two different forms of CGFs on day 14. (B) The mineralization capability of MC3T3-E1 cells was assessed for the different groups [(a) the control group, (b) the freeze-dried CGF group and the (c) sustained-release CGF group] using Alizarin Red-S (ARS) staining. (d) Result of ARS in samples quantified by $10 \%$ cetylpyridinium chloride. (C) The ALP staining and ARS staining of two forms of CGF at the same time. The first row (a-1 to c-1) is the ALP staining and the second row (a-2 to c-2) is the ARS staining with the same sample in the first row. (a) Control group, (b) freeze-dried CGF group and (c) sustained-release CGF group. ${ }^{* *} \mathrm{P}<0.01,{ }^{* * *} \mathrm{P}<0.001$. CGFs, concentrated growth factors.

biocompatibility and had low cytotoxicity and that the cells could maintain normal growth on the gel. Under scanning electron microscope, it was observed that the gel possessed high porosity to provide suitable space for cell adhesion and proliferation and to allow the exchange of nutrients and waste (Fig. 2). When the gel and the CGFs powder were integrated, the sustained-release CGFs were obtained. The osteogenesis of the sustained-release CGFs compared to the freeze-dried CGFs was verified. It was found that the freeze-dried CGF group showed a stronger ability to boost the proliferation of
MC3T3-E1 cells during the first 6 days (Fig. 5), and in the first 14 days, ALP protein expression increased. Afterward, the sustained-release CGF group achieved better results (Fig. 6). On day 21, the ALP staining and mineralized nodule staining results of the sustained-release CGF group were significantly better than those of the freeze-dried CGF group (Fig. 6). The expression of $O P G, O P N$, Runx 2 and ALP was also increased (Fig. 7).

To evaluate whether chitosan-alginate composite gel could be used as a carrier for growth factors, we investigated the 
A

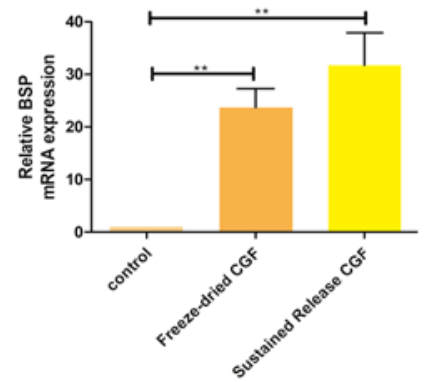

Day 7
B

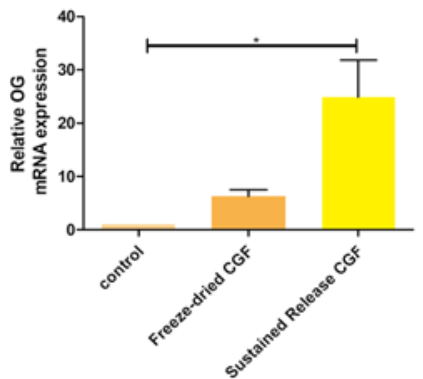

Day 7
C

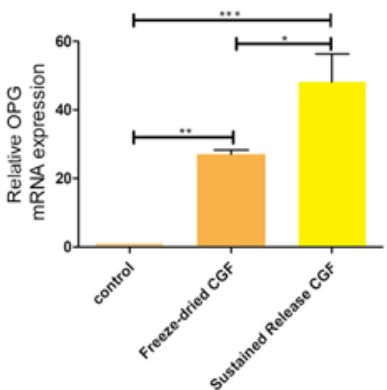

Day 7
D

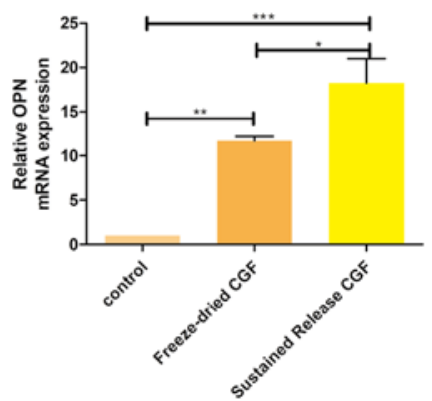

Day 7
E

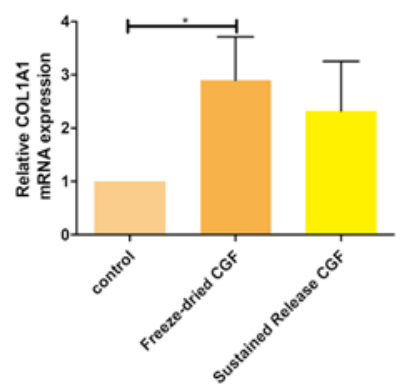

Day 14
$\mathrm{F}$

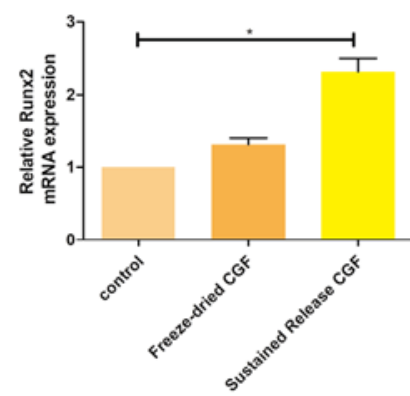

Day 21
G

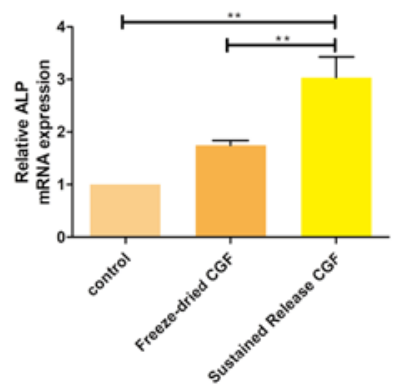

Day 28

Figure 7. Expression of the osteogenesis-associated genes $B S P, O G, O P G, O P N, C O L 1 A 1$, Runx2 and ALP were evaluated by RT-qPCR on days 7, 14, 21, 28 (data not shown for all times). Addition of freeze-dried CGF and sustained-release CGF to the culture media significantly increased expression of (A) $B S P$, (B) $O G$, (C) $O P G$ and (D) $O P N$ in the MC3T3-E1 cells on day 7, (E) COL1A1 on day 14 and (F) Runx2 on day 21. Significant upregulation of (G) ALP was found in the MC3T3-E1 cells cultured with the freeze-dried CGFs and sustained-release CGFs compared to that in the control group on day 28 . "P<0.05, ${ }^{* *} \mathrm{P}<0.01,{ }^{* * *} \mathrm{P}<0.001$. CGFs, concentrated growth factors; $O P G$, osteoprotegerin; $O G$, osteocalcin; Run $x 2$, runt-related transcription factor 2; $B S P$, bone sialoprotein; OPN, osteopontin; ALP, alkaline phosphatase; COL1A1, collagen rype I $\alpha 1$.

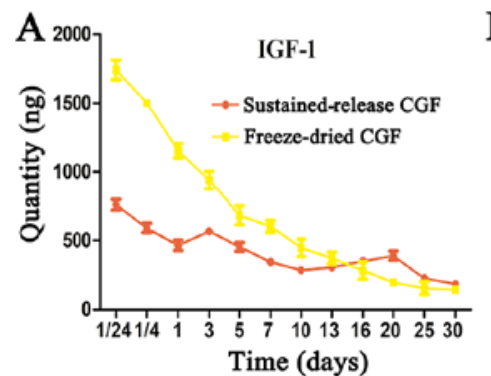

Time (days)

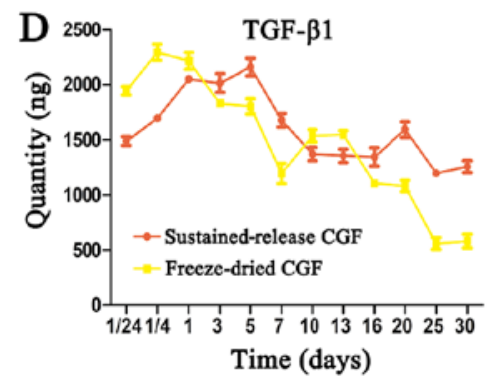

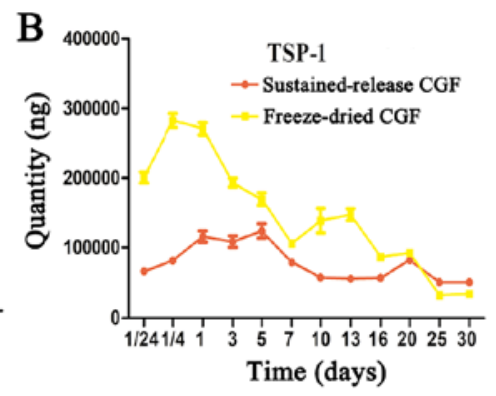
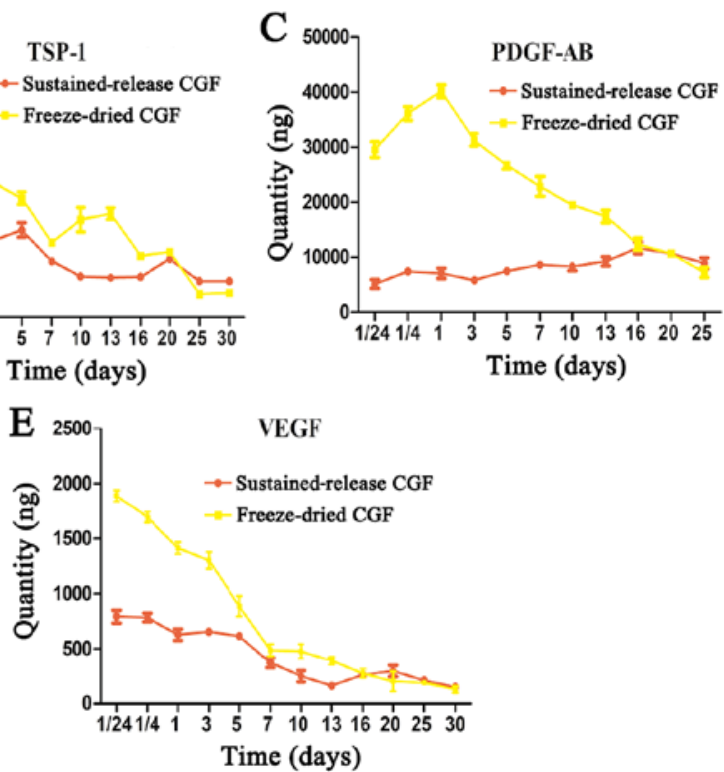

Figure 8. The release of (A) IGF-1, (B) TSP-1, (C) PDGF-AB, (D) TGF- $\beta 1$ and (E) VEGF from the freeze-dried CGFs and the sustained-release CGFs for 30 days. Values are expressed as the cumulative mean quantity of molecules at 1, 6, 24 h, 3, 5, 7, 10, 13, 16, 20, 25 and 30 days. IGF-1, insulin-like growth factor-1; TSP-1, thrombospondin-1; PDGF-AB, platelet-derived growth factor-AB; TGF- $\beta 1$, transforming growth factor $\beta 1$; VEGF, vascular endothelial growth factor; CGFs, concentrated growth factors.

concentration of growth factors released from sustained-release CGF and freeze-dried CGF in vitro were investigated. Our data revealed that different growth factors showed different time-dependencies. 
The thrombospondin-1 protein (TSP-1) has been shown to affect platelet aggregation, angiogenesis, and tissue remodeling $(26,27)$. TSP-1 is a major regulator of transforming growth factor $\beta-1$ (TGF- $\beta 1$ ) activation but also has TGF- $\beta$-independent functions in hemostasis, cell adhesion, migration, and growth factor (EGF, VEGF, FGF) regulation (28). TGF- $\beta 1$ performs many cellular functions, including the control of cell growth, cell proliferation, cell differentiation, and apoptosis (29). TGF- $\beta 1$ increases the synthesis of collagen types I, III and IV and the deposition of fibronectin, proteoglycans and tenascin (30). IGF-I is an important factor that regulates bone cell function and metabolism. It can reduce collagen degradation, increase bone deposition, and promote osteoblast differentiation, maturation and supplementation (31). Platelet-derived growth factor-AB (PDGF-AB) promotes the secretion of collagen and glycoproteins by osteoblasts to synthesize the bone matrix through the action of osteoblasts and participates in bone matrix calcification (32). Vascular endothelial growth factor (VEGF) influences skeletal development and postnatal bone repair. Modulation of VEGF levels in bones represents a potential strategy for treating compromised bone repair and improving bone regeneration (33).

In the present study, at the beginning of the experiment, more IGF-1, VEGF, TGF- $\beta 1$ and PDGF-AB were released by the freeze-dried CGFs than by the sustained-release CGFs. The release rate of these growth factors then decreased rapidly in the freeze-dried CGF group, while the release rate of the sustained release CGF group increased slowly. On day 7, the concentrations of IGF-1 and VEGF in the two groups were almost the same. On day 14, the concentrations of TGF- $\beta 1$ and PDGF-AB in the two groups were almost the same (Fig. 8). This result may be relevant for the different growth factor release patterns of freeze-dried CGFs and sustained-release CGFs. In the freeze-dried CGF group, the growth factors were in a state of free diffusion in the extract, and they were completely exposed to the solution. Over time, the growth factors slowly inactivated; then, the concentration of the growth factors in the solution decreased. In the sustained-release CGF group, the gel carrier enveloped the growth factors so that they were slowly released in the solution, and the concentration of the growth factors was maintained for a long period. However, the pore structure of the gel helped the cells adhere, which benefits cell proliferation and osteogenesis.

After day 14, the concentration of growth factors in the freeze-dried group decreased sharply from that at the beginning, while the sustained-release group maintained a stable concentration, which made the osteogenic expression ability of the sustained-release group significantly higher than that of the freeze-dried group. In the present study, a significant phenomenon was noted. The medium used in the design of this experiment was not supplemented with osteogenic mineralization-inducing solution. However, in both experimental groups, obvious mineralized nodules were observed, which suggests that the composite growth factors in CGFs may have the ability to promote osteoblast self-mineralization. However, this possibility requires verification through further experiments.

Numerous drug delivery systems based on the association of chitosan have been reported in the literature (34). For example, layered composite hydrogels have been used as cell culture carriers and matrices for the control release (35), and microparticles based on alginate/chitosan, alginate-chitosan beads or chitosan-coated alginate beads have been used as sustained release drug delivery systems (36). The results of the present study verified that the use of chitosan-alginate gel carrier can achieve the slow release of growth factors in CGFs and that the sustained-release CGFs can achieve superior osteogenic effects.

In conclusion, the freeze-dried CGFs demonstrated superior osteogenic performance than the sustained-release CGFs in the early stages. Over time, the sustained-release CGFs had significant advantages over the freeze-dried CGFs in terms of promoting osteogenic mineralization. The present study revealed that lyophilization and the chitosan-alginate carrier enabled the growth factors in CGFs to maintain a stable release concentration and to achieve a superior osteogenesis-promotive effect.

\section{Acknowledgements}

Professor Yin Xiao from the Institute of Health and Biomedical Innovation of the Queensland University of Technology of Australia is kindly thanked for providing assistance with the experiments.

\section{Funding}

The study was supported by the Liwan District Science and Technology Project (grant no. 2016080065).

\section{Availability of data and materials}

The datasets used and analyzed during the current study are available from the corresponding author on reasonable request.

\section{Authors' contributions}

LW and MW made equal contributions to this article and should be regarded as co-first authors. LW conceived and designed the study. MW and ZL performed the experiments. MW wrote the initial manuscript. NZ and DL were responsible for data and statistical analyses. LG reviewed the findings and LG checked the acquired data, contributed in the analysis and interpretation of data and edited the manuscript. All authors read and approved the manuscript and agree to be accountable for all aspects of the research in ensuring that the accuracy or integrity of any part of the work are appropriately investigated and resolved.

\section{Ethics approval and consent to participate}

The collection and use of blood samples were performed with informed consent from the volunteers and all protocol consistently adhered to the privacy rights of the human volunteers. This study was approved by the Ethics Committee of Stomatology Hospital, Guangzhou Medical University (KY2018013).

\section{Patient consent for publication}

Not applicable. 


\section{Competing interests}

The authors declare no conflict of interest associated with the present study.

\section{References}

1. Whitman DH, Berry RL and Green DM: Platelet gel: An autologous alternative to fibrin glue with applications in oral and maxillofacial surgery. J Oral Maxillofac Surg 55: 1294-1299, 1997.

2. de Vos RJ, Weir A, van Schie HT, Bierma-Zeinstra SM, Verhaar JA, Weinans $\mathrm{H}$ and Tol JL: Platelet-rich plasma injection for chronic Achilles tendinopathy: A randomized controlled trial. JAMA 303: 144-149, 2010.

3. Dohan DM, Choukroun J, Diss A, Dohan SL, Dohan AJJ, Mouhyi J and Gogly B: Platelet-rich fibrin (PRF): A secondgeneration platelet concentrate. Part III: Leucocyte activation: A new feature for platelet concentrates? Oral Surg Oral Med Oral Pathol Oral Radiol Endod 101: e51-e55, 2006.

4. Anitua E, Aguirre JJ, Algorta J, Ayerdi E, Cabezas AI, Orive G and Andia I: Effectiveness of autologous preparation rich in growth factors for the treatment of chronic cutaneous ulcers. J Biomed Mater Res B Appl Biomater 84: 415-421, 2008.

5. Rodella LF, Favero G, Boninsegna R, Buffoli B, Labanca M, Scarì G, Sacco L, Batani T and Rezzani R: Growth factors, CD34 positive cells, and fibrin network analysis in concentrated growth factors fraction. Microsc Res Tech 74: 772-777, 2011.

6. Kawase $\mathrm{T}$ and Tanaka T: An updated proposal for terminology and classification of platelet-rich fibrin. Regen Ther 7: 80-81, 2017.

7. Sohn DS, Moon JW, Moon YS, Park JS and Jung HS: The use of concentrated growth factor (CGF) for sinus augmentation. J Oral Implant 38: 25-38, 2009.

8. Sohn DS, Heo JU, Kwak DH, Kim DE, Kim JM, Moon JW, Lee JH and Park IS: Bone regeneration in the maxillary sinus using an autologous fibrin-rich block with concentrated growth factors alone. Implant Dent 20: 389-395, 2011.

9. Yu B and Wang Z: Effect of concentrated growth factors on beagle periodontal ligament stem cells in vitro. Mol Med Rep 9: 235-242, 2014

10. He L, Lin Y, Hu X, Zhang Y and Wu H: A comparative study of platelet-rich fibrin (PRF) and platelet-rich plasma (PRP) on the effect of proliferation and differentiation of rat osteoblasts in vitro. Oral Surg Oral Med Oral Pathol Oral Radiol Endod 108 707-713, 2009.

11. Bhattarai N, Gunn J and Zhang M: Chitosan-based hydrogels for controlled, localized drug delivery. Adv Drug Deliv Rev 62: 83-99, 2010.

12. Riva R, Ragelle H, des Rieux A, Duhem N, Jérôme C and Préat V: Chitosan and chitosan derivatives in drug delivery and tissue engineering. Adv Polym Sci 244: 19-44, 2011.

13. Sonia TA and Sharma CP: Chitosan and its derivatives for drug delivery perspective. Adv Polym Sci 243: 23-53, 2011.

14. Mengatto LN, Helbling IM and Luna JA: Recent advances in chitosan films for controlled release of drugs. Recent Pat Drug Deliv Formul 6: 156-170, 2012.

15. Kumar AS and Ramaswamy NM: Chitosan microspheres as potential vaccine delivery systems. Int J Drug Deliv 3: 43-50, 2011.

16. Taşkın AK, Yaşar M, Ozaydın I, Kaya B, Bat O, Ankaralı S Yildırım U and Aydın M: The hemostatic effect of calcium alginate in experimental splenic injury model. Ulusal Travma Acil Cerrahi Derg 19: 195-199, 2013.

17. Pinkas $\mathrm{O}$ and Zilberman M: Effect of hemostatic agents on properties of gelatin-alginate soft tissue adhesives. J Biomater Sci Polym Ed 25: 555-573, 2014.

18. He Q, Gong K, Ao Q, Ma T, Yan Y, Gong Y and Zhang X: Positive charge of chitosan retards blood coagulation on chitosan films. J Biomater Appl 27: 1032-1045, 2013.

19. Lam PL, Lee KK, Wong RS, Cheng GY, Cheng SY, Yuen MC, Lam KH, Gambari R, Kok SH and Chui CH: Development of hydrocortisone succinic acid/and 5-fluorouracil/chitosan microcapsules for oral and topical drug deliveries. Bioorg Med Chem Lett 22: 3213-3218, 2012.
20. Prins HJ, Braat AK, Gawlitta D, Dhert WJ, Egan DA, Tijssen-Slump E, Yuan H, Coffer PJ, Rozemuller $H$ and Martens AC: In vitro induction of alkaline phosphatase levels predicts in vivo bone forming capacity of human bone marrow stromal cells. Stem Cell Res 12: 428-440, 2014.

21. Casati MZ, de Vasconcelos Gurgel BC, Gonçalves PF, Pimentel SP, da Rocha Nogueira Filho G, Nociti FH Jr and Sallum EA: Platelet-rich plasma does not improve bone regeneration around peri-implant bone defects - a pilot study in dogs. Int J Oral Maxillofac Surg 36: 132-136, 2007.

22. Schaaf H, Streckbein P, Lendeckel S, Heidinger KS, Rehmann P, Boedeker RH and Howaldt HP: Sinus lift augmentation using autogenous bone grafts and platelet-rich plasma: Radiographic results. Oral Surg Oral Med Oral Pathol Oral Radiol Endod 106: 673-678, 2008

23. Dohan Ehrenfest DM, de Peppo GM, Doglioli $P$ and Sammartino G: Slow release of growth factors and thrombospondin-1 in Choukroun's platelet-rich fibrin (PRF): A gold standard to achieve for all surgical platelet concentrates technologies. Growth Factors 27: 63-69, 2009.

24. Marx RE: Platelet-rich plasma (PRP): What is PRP and what is not PRP? Implant Dent 10: 225-228, 2001.

25. Park HC, Kim SG, Oh JS, You JS, Kim JS, Lim SC, Jeong MA, Kim JS, Jung C, Kwon YS and Ji H: Early bone formation at a femur defect using CGF and PRF grafts in adult dogs: A comparative study. Implant Dent 25: 387-393, 2016.

26. Lu Z and Kipnis J: Thrombospondin 1-a key astrocyte-derived neurogenic factor. FASEB J 24: 1925-1934, 2010.

27. Zhao C, Isenberg JS and Popel AS: Human expression patterns: Qualitative and quantitative analysis of thrombospondin-1 under physiological and pathological conditions. J Cell Mol Med 22: 2086-2097, 2018.

28. Murphy-Ullrich JE and Suto MJ: Thrombospondin-1 regulation of latent TGF- $\beta$ activation: A therapeutic target for fibrotic disease. Matrix Biol 68-69: 28-43, 2018

29. Ghadami M, Makita Y, Yoshida K, Nishimura G, Fukushima Y, Wakui K, Ikegawa S, Yamada K, Kondo S, Niikawa N and Tomita Ha: Genetic mapping of the Camurati-Engelmann disease locus to chromosome 19q13.1-q13.3. Am J Hum Genet 66: $143-147,2000$.

30. Le Bousse-Kerdilès MC and Martyré MC: Dual implication of fibrogenic cytokines in the pathogenesis of fibrosis and myeloproliferation in myeloid metaplasia with myelofibrosis. Ann Hematol 78: 437-444, 1999.

31. Wang CY, Li XD, Hao ZH and Xu D: Insulin-like growth factor-1 improves diabetic cardiomyopathy through antioxidative and anti-inflammatory processes along with modulation of Akt/GSK-3 $\beta$ signaling in rats. Korean J Physiol Pharmacol 20: 613-619, 2016.

32. Pierce GF, Tarpley JE, Yanagihara D, Mustoe TA, Fox GM and Thomason A: Platelet-derived growth factor (BB homodimer), transforming growth factor-beta 1 , and basic fibroblast growth factor in dermal wound healing. Neovessel and matrix formation and cessation of repair. Am J Pathol 140: 1375-1388, 1992.

33. Hu K and Olsen BR: The roles of vascular endothelial growth factor in bone repair and regeneration. Bone 91: 30-38, 2016.

34. Liu Y, Chen L, Zhou C, Yang J, Hou Y and Wang W: Development and evaluation of alginate-chitosan gastric floating beads loading with oxymatrine solid dispersion. Drug Dev Ind Pharm 42: 456-463, 2016

35. He M,Zhang X, Yao W, Wang C, Shi L and Zhou P: Construction of alternate layered chitosan/alginate composite hydrogels and their properties. Mater Lett 200: 43-46, 2017.

36. Coppi G and Iannuccelli V: Alginate/chitosan microparticles for tamoxifen delivery to the lymphatic system. Int J Pharm 367: 127-132, 2009.

This work is licensed under a Creative Commons Attribution-NonCommercial-NoDerivatives 4.0 International (CC BY-NC-ND 4.0) License. 